\title{
Mögliche Auswirkungen der Einführung von DRGs auf vulnerable Patientengruppen
}

\author{
Eine Studie im Rahmen der Begleitforschung soll die Auswirkungen der DRGs auf \\ die Behandlung und Betreuung älterer und schwerkranker Patienten vor und nach \\ Spitalaufenthalt klären. Der erste Teil der Untersuchung zeigt die Befragungsergeb- \\ nisse vor der Einführung der DRGs. Dieselben Fragen werden in eineinhalb Jahren \\ erneut gestellt.
}

Markus Breuer,

Ruth Baumann-Hölzle

Die hier vorgestellte Studie ist als Download unter www.dialogethik.ch verfügbar und beim Institut Dialog Ethik zum Selbstkostenpreis zu beziehen.

Korrespondenz:

Dr. oec. Markus Breuer

Dr. theol.

Ruth Baumann-Hölzle

Institut Dialog Ethik

Schaffhauserstrasse 418

CH-8050 Zürich

Tel. 0442524201

mbreuer[at]dialog-ethik.ch

\section{Vulnerable Patientengruppen}

Die Diskussionen über das schweizerische Gesundheitssystem, die jährlichen Debatten über den Prämienanstieg sowie der demografische Wandel lassen verstärkt die Frage aufkommen, was wir uns noch leisten können und ob das Preis-LeistungsVerhältnis im Gesundheitswesen angemessen ist. Unter diesem Kostendruck ist auch die Einführung der DRGs zu sehen, die 2012 erfolgen wird. Diese Veränderung ist, wie in der Schweizerischen Ärztezeitung bereits vielfach debattiert, nicht unumstritten. Die im Ausland gemachten Erfahrungen im Hinblick besonders auf vulnerable Patientengruppen sind ambivalent. Die Auswirkungen können gerade auf die Schwächsten - alte und gebrechliche Menschen, Menschen mit Behinderung usw. - negativ sein [1].

«Gelingt es nicht, die Behandlung der Schwerstkranken nach solidarischen und sozialen Kriterien zu gewährleisten, dann ist ein entscheidendes Zielkriterium nicht erfüllt. Die Gruppe der Schwerstkranken hat aufgrund von Verdrängungsmechanismen eine denkbar schlechte Lobby. Die Behandlung dieser Patienten erfordert in einem stark ökonomisch ausgerichteten Gesundheitswesen besondere Aufmerksamkeit.» [2]

Vor dem Hintergrund dieser Erfahrungen und der mangelnden Lobby für vulnerable Patientengruppen sind die Auswirkungen der DRG-Einführung auf ältere und gebrechliche Menschen besonders $\mathrm{zu}$ prüfen.

\section{Der besondere Auftrag eines Spitals}

Was führt zu einer Spitaleinweisung älterer Menschen? Neben den rein medizinischen Gründen gibt es psychosoziale Gründe für die Einweisung, zum Beispiel «Versorgungsprobleme, durch Überlastung des sozialen Netzes, der Ausfall der bisherigen Pflegeperson oder ein plötzlich höherer Hilfebedarf» (3). Die Spitaleinweisung lässt sich auch in diesen Situationen medizinisch begründen, aber sie wird in erster Linie aus psychosozialen Grün-

\section{Conséquences possibles de} l'introduction des DRG sur des groupes de patients vulnérables

Dans quelle mesure, l'introduction des forfaits par cas influe-t-elle sur le traitement et la prise en charge des groupes de patients vulnérables, tels que les malades chroniques ou les personnes âgées? Une étude concomitante de l'Institut Dialog Ethik entend répondre à cette question. Il s'agit d'analyser les conséquences des DRG sur le traitement et la prise en charge des patients avant et après leur hospitalisation. Quel sera l'impact de l'introduction des forfaits par cas sur les traitements prodigués par les médecins de famille, sur les soins à domicile et sur la prise en charge dans des homes médicalisés? Cette étude pose surtout la question de savoir quel sera le rôle des facteurs psychosociaux au moment de l'admission du patient à l'hôpital et de sa sortie avant et après l'introduction des DRG. Cet article présente les résultats d'une enquête effectuée avant l'introduction des DRG. Les mêmes questions seront reposées dans une année et demie. Les deux enquêtes seront ensuite comparées afin de tirer des conclusions sur les effets concrets des DRG.

Les questions ont été élaborées sur la base des activités de l'Institut Dialog Ethik dans différents hôpitaux et établissements de soins de longue durée. L'institut accompagne ces institutions lors du développement, de l'organisation et de la mise en œuvre des systèmes de soutien éthique. Dans ce contexte, il a pu se familiariser directement avec les attentes et les craintes des collaborateurs et des patients avant I'introduction des forfaits par cas. 
den erwogen. «Es (das Spital - Anm. d. Verf.) fängt Patienten auf, die aufgrund infrastruktureller Defizite der ambulanten Versorgung und durch sie verursachter Diskontinuitäten nicht zu Hause versorgt werden können, kompensiert die zu eng gesteckten Leistungsgrenzen anderer Versorgungseinrichtungen, springt ein, wenn sich Versorgungsarrangements als nicht tragfähig erweisen und zusammenbrechen (...) und schliesst Versorgungslücken.» [4]

Die Gesundheitswissenschaftlerin Doris Schaeffer (Universität Bielefeld) spricht von einer Substitutionsfunktion des Spitals. Wird die Institution Spital dieser Funktion nach Einführung der DRGs noch angemessen gerecht werden können? «Es erfolgt eine schrittweise Abkehr von der psychosozialen Funktion der Institution Krankenhaus, hin zu einem rein (akut) medizinischen, somatisch orientierten Aufgabenverständnis. Diese Veränderung (...) führt dazu, dass insbesondere ältere, chronisch kranke Patienten als (fehlplatziert) wahrgenommen werden und unter-
Systems und nach einer gewissen Zeitspanne je eine qualitative Befragung der «zugewandten Dienste» (Hausärzte, Spitex, Heime) durchgeführt. Vorliegend haben wir es mit den Befragungsergebnissen vor der Einführung der DRGs zu tun.

\section{Hauptresultate der Studie vor der Einführung der DRGs}

Vulnerable Patienten benötigen zwei- bis dreimal so viel Zeit wie andere Patienten. Die «zugewandten Dienste» sind der Überzeugung, dass sie über das medizinisch-fachliche Know-How für die Bedürfnisse dieser Patientengruppe verfügen. Lediglich in Bezug auf die sogenannten «ethisch-menschlichen Anforderungen» sowie die zeitlichen Ressourcen werden Einschränkungen gemacht. Aber auch die zeitliche Kapazität der Spitäler wird von den meisten Befragten als kritisch betrachtet. Dort sei der Spardruck besonders gross, was dazu führe, dass pro Patient weniger Zeit aufgewendet werden könne. Dies läuft den Bedürfnissen von vulne-

\section{«Die Gruppe der Schwerstkranken hat aufgrund von Verdrängungs- mechanismen eine denkbar schlechte Lobby.»}

schiedlichsten Externalisierungsstrategien ausgesetzt sind - bei gleichzeitigen Mängeln im komplementären Versorgungssystem der Altenhilfe.» [5]

Die Rolle des Spitals könnte sich mit Einführung der DRGs grundlegend verändern [6]. «Diese Veränderungen widersprechen teilweise dem bisherigen Selbstverständnis der betroffenen Professionen und können zu Spannungen führen zwischen dem therapeutischen Ethos, Hilfe zu leisten, und der neuen Aufgabe, Krankheitswertigkeiten zu dokumentieren und berechnend ins ärztliche und pflegerische Handeln einfliessen zu lassen.» [7]

\section{Das Forschungsprojekt «Vulnerable Gruppen und DRGs"}

Das Institut Dialog Ethik führte in den Kantonen Zürich, Bern, Basel-Stadt und Baselland gemeinsam mit der gfs-Zürich eine qualitative Begleitforschung zur Einführung der DRGs durch. Es ging um die Fragen der Auswirkungen der DRGs auf Behandlung und Betreuung älterer Patienten vor und nach Spitalaufenthalt. Wie wirkt sich die Einführung der Fallkostenpauschalen auf die Behandlung der Hausärzte aus, auf die Betreuung der Spitex sowie auf die Behandlung und Betreuung in den Alters- und Pflegeheimen? Welche Rolle spielen psychosoziale Faktoren bei Spitaleintritt und Spitalaustritt vor und nach der Einführung?

Um die befürchteten und effektiven Auswirkungen zu erfassen, wird vor der Einführung des neuen rablen Patienten zuwider, da diese eher mehr Zeit benötigen.

Die Befragten äusserten sich positiv über die derzeitige Möglichkeit, Spitaleinweisungen auch mit psychosozialen Gründen erwirken zu können. Auch mit dem Austrittszeitpunkt aus dem Spital waren die Befragten grundsätzlich zufrieden. Allerdings werden bereits heute eine bessere Absprache sowie eine frühzeitigere Information gewünscht. Auf psychosoziale Aspekte, beispielsweise ob eine ausreichende anschliessende Betreuung gewährleistet ist, werde nicht immer genügend Rücksicht genommen.

\section{«Vulnerable Patienten benötigen zwei- bis dreimal so viel Zeit wie andere Patienten.»}

\begin{abstract}
Ausblick auf die DRGs - Chancen und Risiken
Die Mehrheit der Befragten verbindet mit dem neuen System Hoffnung auf Verbesserungen im Patientenwesen. Ein Heim in Zürich hat sich bereits auf den Systemwechsel eingestellt und betrachtet das neue System als flexibler als das alte. Die Kostenfrage sei klarer geregelt (Betreuung und Pflege werden klar getrennt: die Betreuung zahlt der Patient, den Aufenthalt zahlt die Gemeinde,
\end{abstract}


Wie werden sich frühere Spital-Entlassungen auf ältere und chronisch kranke Patienten auswirken? Einige Befragte befürchten den «Drehtür-Effekt», andere sehen eine Chance, dass Patienten verloren geglaubte Ressourcen und Lebensqualität wieder erlangen, wenn sie sich zu Hause erholen können.

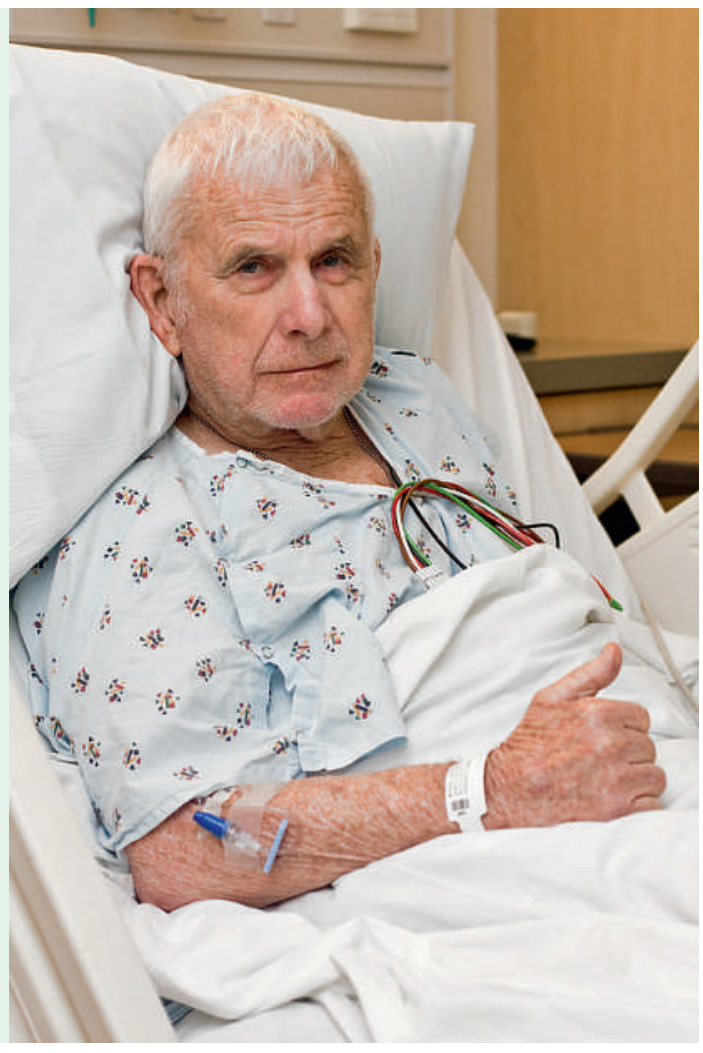

Medizinisches zahlt die Krankenkasse). Es sei ihnen neu möglich, auch Patienten mit vielen Medikamenten aufzunehmen. In der bedarfsgerechten und transparenten Finanzierung sehen die meisten die grössten Chancen der Umstellung. Ausserdem erhofft man sich:

- sinkende Kosten, ausgeglichenere Kosten pro Fall;

- effizientere Patientenwege
Als beispielhaft kann vielleicht die Aussage einer Befragten genannt werden, die ihre Hoffnungen in Bezug auf die Neuausrichtung so formuliert: «Wenn das System funktionieren soll, muss man mehr Zeit für Gespräche mit den Patienten einräumen, damit wirklich nur diejenigen Behandlungen gemacht werden, die zum Wohl des Patienten sind. Ohne genügende frühzeitige Abklärung wird es für die Spitäler mit dem neuen System teurer, und so hoffe ich, dass dies zu einer höheren Sensibilisierung für die Konsequenzen des eigenen Handelns bei den verschiedenen Akteuren führt.»

Die Befragten sehen im neuen System auch Risiken. Häufig stehen diese in Zusammenhang mit den Punkten, die als positive Faktoren der heutigen Regelung genannt wurden:

- Individualität geht verloren;

- übermässiger Zeitdruck;

- nicht mehr in jedem Fall adäquate Behandlung;

- Schnittstellen könnten leiden, wenn es immer schneller gehen muss.

Die Befürchtung ist gross, dass die Patienten nur noch als reiner Kostenfaktor wahrgenommen werden, dass die Ökonomie dermassen im Vordergrund steht und dass das Wohlbefinden der Patienten zu kurz komme. Gerade bei komplexen Patienten bestehe die Gefahr, dass man ihnen nicht mehr gerecht werde. Es wird zudem befürchtet, dass weniger auf den Allgemeinzustand, weniger auf das soziale Netzwerk sowie die Anschlusstherapie geachtet wird. Man befürchtet einen Karusselleffekt: Bei zu früher Entlassung wird der Patient bald wieder zurück im Spital sein. Dies sei auf Dauer nicht billiger und für den Patienten sicher nicht nützlich.

\section{«Die Mehrheit der Befragten verbindet mit dem neuen System Hoffnung auf Verbesserungen im Patientenwesen.»}

- sorgfältigere Abklärungen, weil Fehlbeurteilungen teuer werden;

- bessere Vernetzung unter den Stakeholdern;

- Aufwertung der Hausärzte (wenn sie präzise Diagnosen und Nebendiagnosen stellen, bringen sie dem Spital Geld);

- Umdenken im Machbarkeitswahn, weniger «unnötige» Behandlungen;

- Aufdeckung von Lücken im System (werden offensichtlich);

- spannenderes Arbeitsfeld für Spitex (auch Akutbehandlungen);

- frühere Heimkehr der Bewohner ins Heim.
Die einzelnen Akteure haben auch Angst, dass sie gegeneinander ausgespielt werden:

- Hausärzte befürchten, dass eine Lawine an Arbeit auf sie zukomme, die sie nicht bewältigen könnten, zumal es schon jetzt einen Mangel an Hausärzten gebe. Wer übernimmt die Aufgaben, wenn mehr in den ambulanten Bereich übertragen wird?

- Spitex-Mitarbeitende befürchten, zwischen den verschiedenen Kostenfinanzierern zerrieben zu werden.

- Heime und Spitex erwarten neue technische und personelle Herausforderungen für den Fall, dass Patienten früher entlassen werden (Infu- 
sionstherapien), womit sich neue Fragen $\mathrm{zu}$ ihren Qualifikationen und zur Qualität stellen werden.

Darüber, welche und wie viele Behandlungen ein Patient nach Einführung der DRGs noch erhält, bestehen ebenfalls Unsicherheiten. Es gibt sowohl die Prognose, es werde zukünftig ein Fokus auf die Hauptdiagnose gelegt und Nebendiagnosen würden vernachlässigt, wie auch diejenige, dass mehr bzw. zu viele Diagnosen gestellt und Abklärungen vorgenommen würden, um die Fallpauschalen abholen zu können.

Man ist sich zwar einig, dass die Patienten nach der Umstellung tendenziell früher aus dem Spital entlassen werden; die Meinungen darüber, ob dies nun gut oder schlecht sei, sind aber geteilt. Die einen befürchten dadurch neue Komplikationen oder auch die Verhinderung einer möglichen Rehabilitation; andere sehen darin durchaus eine Chance, dass Patienten bestärkt werden und verloren geglaubte Ressourcen und Lebensqualität wieder erlangen, wenn sie sich zu Hause erholen können.

Zusammenfassend kann festgehalten werden, dass sich im Moment keine klare Tendenz bezüglich Hoffnungen und Ängste bei den Befragten hinsichtlich möglicher Auswirkungen der Fallpauschaleneinführungen auf vulnerable Patientengruppen ausmachen lässt. Man ist sehr gespannt, wie sich diese Systemneuerung tatsächlich auswirken wird. Auf jeden Fall ist humanitäre Wachsamkeit geboten.

\section{Danksagung}

Wir möchten uns für die Unterstützung durch die MBF-Foundation, die Teamco-Foundation, die W. Haefner Stiftung, die Otto Gamma Stiftung sowie den simovita Verein bedanken, ohne die dieses Projekt nicht hätte durchgeführt werden können. Unser Dank gilt auch Herrn Martin Abele und Frau Nina Blumenfeld von der gfs-Zürich.

\section{Referenzen}

1 So zeigt eine Befragung eine zunehmende Verschlechterung des Gesundheitszustandes nach Einführung der DRGs. «Auswirkungen der DRGEinführung auf die Rehabilitation - Eine Befragung von Rehakliniken» (WWU Münster - IKM 2008) oder «Rehabilitation unter Kosten- und Qualitätsdruck, Konsequenzen der DRG-Einführung für Patienten und Versorgungsstruktur» (Eiff, Universität Münster) «Bezüglich der innerhalb von 90 Tagen nach einer Krankenhausentlassung erfolgten Wiedereinweisung ins Krankenhaus. Diese erfolgte in DRG-Krankenhäusern beträchtlich häufiger als in Nicht-DRG-Kliniken.» In: Busato A und von Below G. The implementation of DRG-based hospital reimbursement in Switzerland: A population-based perspective. Health Research Policy and Systems. 2010; 8:31; doi 10.1186/1478-4505-8-31. Auch B. Braun (Der Einfluss der DRG-Einführung auf die Situation der Patientinnen und Patienten in Deutschland), Zentrum für Sozialpolitik, Universität Bremen, kommt für die Situation der Patientinnen und Patienten in Deutschland zu dem Ergebnis, dass eher die Befürchtungen erkennbar eintreffen als die Verbesserungen. www.bag.admin.ch/nek-cne/06238/06651/ index.html?lang=it...

2 Billing A. Fallpauschalensystem: Problem Schwerstkranke. In: Deutsches Ärzteblatt. 2005; 102(33) abgerufen 5. Oktober 2011. www.aerzteblatt.de/ v4/archiv/artikel.asp?id=48004

3 Schilling A. Ältere Menschen im Krankenhaus. Kasseler Gerontologische Schriften Band 2. Kassel; 2003. S. 15

4 Schaeffer D. Bruchstellen in der Versorgung chronisch kranker alter Menschen: Die Entlassung aus dem Krankenhaus. In: Seidl E et al. (Hrsg.). Autonomie im Alter. Wien; 2000. S.11-35, zit. n. Schilling A [3].

5 Schilling A [3].

6 Vgl. Baumann-Hölzle, R. Von der Heilpflege zur Gesundheitswerkstatt - von der Heilsökonomie zum Gesundheitsmarkt. PrimaryCare. 2010;(17):324-5.

7 Zur Einführung von diagnosebezogenen Fallpauschalen in Schweizer Spitälern. Stellungnahme der NEK/ CNE Nr. 15/2008. S.1534 f. 